\title{
Vigilancia epidemiológica del síndrome de Down en Chile, 1972 a 2005
}

\author{
Julio $\mathbf{N}$ azer $\mathbf{H}^{1}$, Alfredo Aguila $\mathbf{R}^{1}$, Lucía Cifuentes $\mathbf{0}^{2}$. \\ Increasing rates of Down syndrome \\ among newborns in Chile \\ from 1972 to 2005
}

Background: There are some records shrowing that the frequency of Down syndrome is experiencing an increase over time. Aim: To verify whether the frequency of Down syndrome is increasing in the maternity of the University of Chile Clinical Hospital, or in other Chilean hospitals participating in the Latin American Collaborative Study of Congenital Malformations (ECLAMC) and compare the rates with other world registries of congenital malformations. Material and methods: The information was obtained from the ECLAMC databases of the maternity. The Down syndrome incidence rates were calculated from 1997 to 2005 and rate curves were constructed. Results: The overall rate of Down syndrome was 3.36 per 10,000 born alive. This rate experienced a significant increase in the study period. These rates differ in the different Chilean regions, being higher in Santiago and lower in the Southern regions of the country. The mean age of mothers of newborns with or without Down syndrome was $36 \pm 6$ and $29 \pm 6$ years, respectively, $p>0.001$. Conclusions: The rates of newborns with Down syndrome increased in the period 1972-2005, bearing a close relationship with the increase in maternal ages (Rev Méd Chile 2006; 134: 1549-57).

(Key words: Down syndrome; Maternal age; Nervous system malformations)

Recibido el 5 de octubre, 2005. Aceptado el 21 de abril, 2006.

${ }^{1}$ Unidad de Neonatología, Hospital Clínico Universidad de Chile. ${ }^{2}$ Programa de Genética Humana, Instituto de Ciencias Biomédicas, Facultad de Medicina, Universidad de Chile. Santiago de Chile.

$\mathrm{E}^{1}$ Hospital Clínico de la Universidad de Chile (HCUCH) inició un registro de malformaciones congénitas, como miembro del Estudio Colaborativo Latinoamericano de Malformaciones Congénitas (ECLAMC) ${ }^{1}$, en septiembre de 1969.

Correspondencia a: Dr. Julio Nazer H. Unidad de Neonatología, Hospital Clínico Universidad de Chile. Av. Santos Dumont 999. Santiago, Chile. Fono: 2774106.
Desde esa fecha se ha mantenido en forma ininterrumpida y a cargo del mismo médico coordinador. La función principal del ECLAMC es monitorizar las frecuencias con que se presentan las malformaciones en general y de cada una en particular. Hemos comunicado nuestros hallazgos, especialmente en esta Revista, en decenas de trabajos. Hemos observado que muchos defectos congénitos han aumentado su prevalencia al nacimiento, especialmente aquellos diagnósticos 
dependientes de la ecografía prenatal, como uropatías obstructivas, cardiopatías congénitas y otras malformaciones internas, que antes de la ecografía se descubrían más tardíamente. Sin embargo, hay otras, como el síndrome de Down, que por lo general no se diagnostica antenatalmente, aun cuando hay signos ultrasonográficos que pueden hacerlo sospechar, que pareciera que están aumentando su frecuencia. En Chile, de acuerdo a los datos publicados, la prevalencia al nacimiento del síndrome de Down está, en la actualidad, por sobre 2,2 por 1.000 nacimientos $^{2}$. Esta tendencia la hemos comunicado en trabajos anteriores ${ }^{3-5} \mathrm{y}$ hemos confirmado la correlación que existe con el aumento del promedio de edad materna en nuestra maternidad ${ }^{6}$. El ECLAMC da, para el período 1982-2001, una tasa de 1,63 por mil (5.841/3.574.609) $)^{7}$.

Se ha relacionado al síndrome de Down con factores de riesgo tan variados como agentes genéticos, físicos, químicos, inmunológicos, infecciosos y sociales, pero solamente tres se consideran en la actualidad como probables agentes etiológicos de la trisomía 21, ellos son: anomalías cromosómicas de los padres capaces de inducir una no-disyunción meiótica secundaria, la exposición precigótica materna a radiaciones ionizantes y la edad materna avanzada. Las dos primeras, si bien son importantes, son responsables, en la práctica, de una minoría de casos de síndrome de Down, mientras que se ha demostrado que existe una estrecha relación entre esta afección y la edad materna $^{5}$. Un tercio de los niños afectados con trisomía 21 nacen de madres de 40 años o más.

Por la importancia médica y sobre todo social, es que ha sido una preocupación permanente de los diferentes programas de registro y monitoreo dedicados a estudiar este problema. Esto nos ha motivado a estar siempre alertas a cualquier cambio que se pueda producir en las prevalencias al nacimiento de esta trisomía y publicando nuestros hallazgos, con el fin de crear conciencia tanto en los médicos, funcionarios de la salud, como en el público en general, de los factores de riesgos que influyen en la aparición de nuevos casos. En repetidas ocasiones hemos comunicado nuestra experiencia y la de otros autores al respecto ${ }^{2-5}$, con el fin de educar y advertir de estos peligros. El ECLAMC publicó un Decálogo para la prevención primaria de las malformacio- nes congénitas y en el № 2 propone que la familia debe completarse mientras se es joven ${ }^{6}$. La frecuencia del síndrome de Down en los diferentes países o en diferentes regiones de un mismo país, puede verse afectada por la diferente distribución de las edades maternas, pero también por el diagnóstico prenatal seguido de aborto electivo, en los países en que está legalmente permitido. De ahí que haya diferencias importantes al compararlos. Sin embargo, esta tendencia al aumento se ha visto en programas de países como Italia, Francia Central-Este, en los que se registra tanto los nacidos vivos como los productos de estos abortos selectivos ${ }^{7}$.

Objetivos del estudio. El objetivo principal de este trabajo es comprobar si existe un aumento de la prevalencia al nacimiento del síndrome de Down.

Establecer las tasas de prevalencia al nacimiento del síndrome de Down en la maternidad del HCUCH en el período 1998-2005.

Comparar estas tasas con las de los otros hospitales chilenos participantes en el ECLAMC y con otros programas mundiales de registro de malformaciones congénitas.

Comparar estas tasas anuales con las de los períodos anteriores estudiados.

Construir una curva de estas tasas anuales del período en estudio, prolongando la ya construida del período anteriormente estudiado, 1972-19975.

Correlacionar las tasas anuales del síndrome de Down con los promedios de edades maternas del total de nacimientos ocurridos en la maternidad.

Evaluar la edad materna como factor de riesgo para síndrome de Down.

Estudiar otros factores de riesgo para tener hijos con síndrome de Down, como antecedentes familiares de otros niños afectados, estudios genéticos realizados.

\section{PACIENTES Y MÉTODO}

Para los efectos de esta revisión, se tomaron los casos acumulados en la base de datos que el ECLAMC tiene en la maternidad. Todos los recién nacidos son examinados por un pediatra neonatólogo del Servicio de Neonatología de la maternidad. A este Programa ingresan todos los recién nacidos 
vivos o mortinatos que tengan un peso de 500 gramos o más y que presenten una o más malformaciones congénitas; se les completa una ficha confeccionada ad hoc según el Manual Operacional del ECLAMC $^{7}$. Se toma como control al recién nacido vivo (RNV), del mismo sexo y sin malformaciones congénitas, que nace a continuación.

El diagnóstico se hizo siguiendo los criterios dados por el ECLAMC en su Manual Operacional, que incluye la especificación de 11 signos clínicos en el examen físico: hipotonía muscular generalizada, laxitud articular, piel redundante de la nuca, hendiduras palpebrales oblicuas, epicantus palpebral, orejas displásticas, protrusión lingual, pliegue palmar único transverso, incurvación radial del dedo meñique y diástasis entre el primer y segundo dedos de los pies. A todo paciente afectado se le efectuó cariograma.

El período estudiado comprende todos los nacimientos ocurridos en la maternidad del Hospital Clínico de la Universidad de Chile, entre el 1 de enero de 1997 y el 30 de junio de 2005.

Para la construcción de la curva de tasas anuales de síndrome de Down, se agregarán los datos obtenidos en el estudio anterior, realizado del período enero de 1972 al 30 de junio de $1997^{5}$.

Se comparó el promedio de edad materna y de peso de nacimiento de los casos con síndrome de Down, con un grupo de 301 recién nacidos controles no malformados elegidos aleatoriamente entre todos los recién nacidos controles registrados durante el período estudiado.

Para comparar nuestras tasas con las del resto de los hospitales chilenos participantes del ECLAMC, contamos sólo con los datos desde 1982 hasta 2001, datos publicados en la versión electrónica del Documento final de la RAE $34^{8}$.

El estudio estadístico se realizó con la prueba de t de Student para comparar promedios de edad materna y peso de nacimiento y prueba de $\chi^{2}$ para comparar las tasas entre los distintos peńodos. Se construyó la recta mínimo cuadrática de la prevalencia al nacimiento del síndrome de Down con el promedio de edad matema como variable predictora.

Se calculó las tasas de prevalencia al nacimiento del síndrome de Down, en forma global y separadamente para nacidos vivos y mortinatos.

\section{RESULTADOS}

En el período 1997-2005 ocurrieron 18.005 nacimientos consecutivos, de los que 17.875 fueron nacidos vivos $(\mathrm{NV})$ y 130 mortinatos $(\mathrm{NM})$. Esto representa una mortinatalidad global de $0,72 \%$. En el total de nacimientos se encontró $1.491 \mathrm{RN}$ malformados (8,28\%), $1.463 \mathrm{NV}(8,18 \%)$ y $28 \mathrm{NM}$ $(21,54 \%)$ (Tabla 1$)$.

Tabla 1. Total de nacimientos, RN V, NM, total de malformados, M FV, M FM y tasas de malformaciones, M FV y M FM , por año desde 1997 a 2005. M aternidad del H ospital Clínico Universidad de Chile

\begin{tabular}{|lrrrrrrrrr|}
\hline & \multicolumn{2}{c}{ Nacimientos } & \multicolumn{3}{c}{ Malformados } & \multicolumn{3}{c|}{ Tasas \% } \\
& Total & NV & NM & MFT & MFV & MFM & MFT & MFV & MFM \\
\hline 1997 & 2.485 & 2.466 & 19 & 204 & 200 & 4 & 8,20 & 8,11 & 21,05 \\
1998 & 2.936 & 2.917 & 19 & 190 & 187 & 3 & 6,41 & 6,41 & 15,78 \\
1999 & 2.278 & 2.267 & 11 & 205 & 204 & 1 & 8,99 & 8,99 & 9,09 \\
2000 & 2.219 & 2.208 & 11 & 170 & 168 & 2 & 7,61 & 7,61 & 18,18 \\
2001 & 2.184 & 2.166 & 18 & 181 & 174 & 7 & 8,28 & 8,03 & 38,89 \\
2002 & 1.860 & 1.840 & 20 & 148 & 145 & 3 & 7,97 & 7,88 & 15,00 \\
2003 & 1.721 & 1.708 & 13 & 155 & 151 & 4 & 9,06 & 8,84 & 30,76 \\
2004 & 1.555 & 1.539 & 16 & 147 & 143 & 4 & 9,43 & 9,29 & 25,00 \\
2005 & 767 & 764 & 3 & 91 & 91 & 0 & 11,64 & 11,91 & 0,00 \\
Total & 18.005 & 17.875 & 130 & 1.491 & 1.463 & 28 & 8,28 & 8,18 & 21,54 \\
\hline
\end{tabular}

$\mathrm{NV}$ =nacidos vivos. $\mathrm{NM}=$ mortinatos. $\mathrm{MFT}=$ total malformados. MFV =malformados vivos. MFM $=$ malformados mortinatos. 
En el total de nacimientos se encontró $65 \mathrm{RNV}$ con síndrome de Down (3,36 por mil). No hubo NM afectados.

Al analizar las tasas de prevalencia al nacimiento del síndrome de Down y de los promedios de edad materna desde 1972 a 2005 (Tabla 2 , Figura 1), se puede apreciar un incremento de la frecuencia de esta patología de 1,03 por mil en 1972 a 5,2 por mil en 2005, habiendo algunos años en que esta cifra se sobrepasó, como en 2002 con 5,98 y 2003 con 10,10 por 1.000 (Tabla 2). Si se comparan las tasas de prevalencia al nacimiento de síndrome de Down bianuales, desde 1972-73 hasta 2004-05, se detecta un incremento gradual altamente significativo $\left(\chi^{2}\right.$ $=71,2$ con 16 grados de libertad; $p=0,0000007$ ).

Los promedios de edad materna de los controles aumentaron de 24,13 en 1977, a cifras cercanas a 30 años desde el año 2000. En cambio, los promedios de edad materna de los niños con síndrome de Down se han mantenido estables.

El promedio de edad matema para el período 1997-2005, fue de 35,5 años (desviación estándar 6,46 años) para las madres de los niños con síndrome de Down y de 28,81 años (desviación estándar 6,08 años), para las madres de niños sin malformaciones congénitas ( $t=7,6 ; p=0,0001)$ (Tabla 3).

Al construir una recta mínimo cuadrática de la tasa de prevalencia al nacimiento de síndrome de Down, sobre los promedios de edad materna de la población general atendida en la maternidad del Hospital Clínico de la Universidad de Chile, se obtiene una recta que incrementa en 0,475 por 1.000 la tasa de síndrome de Down por cada año que aumenta el promedio de edad materna (pendiente positiva) y un coeficiente de correlación de Pearson de 0,416 ( $p=0,014$ ) entre ambas variables (Figura 2). El coeficiente de determinación de 0,16 demuestra, sin embargo, que existen, además de la edad materna promedio, otros factores variables explicativos del aumento en la tasa de síndrome de Down, que no se detectan en el presente análisis. Este hecho se corrobora al observar las curvas de la Figura 1, donde al analizar el comportamiento en el tiempo de la edad materna promedio y la tasa de prevalencia

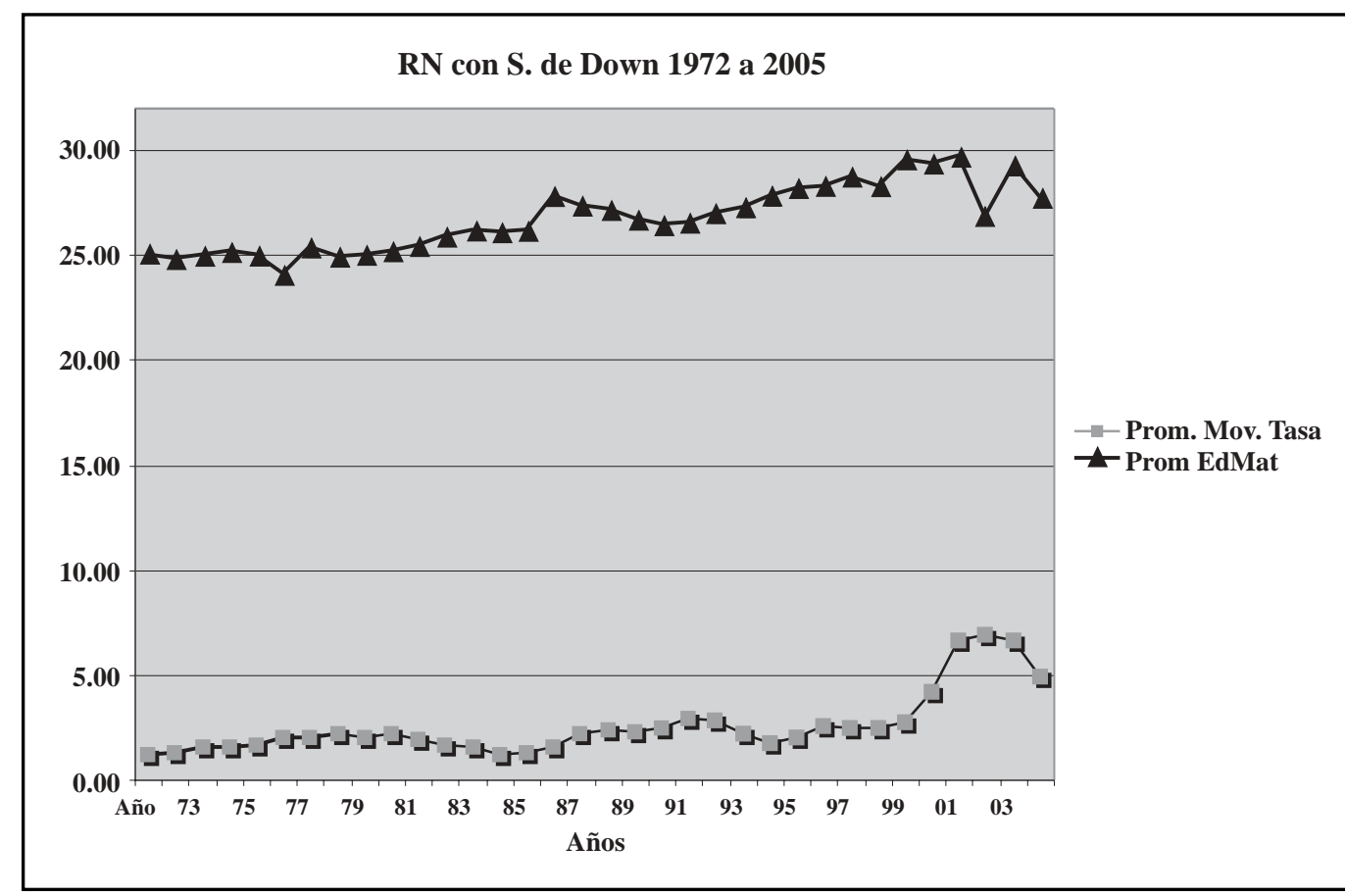

Figura 1. Variación de las tasas de prevalencia de síndrome de Down en relación al promedio de edad materna. Período 1972 a 2005. 
Tabla 2. Tabla histórica de la evolución de las tasas anuales y promedio de edad materna de niños con síndrome de D own y de los promedios de edad materna del total de las madres de la maternidad del H ospital C línico U niversidad de Chile, desde 1972 a 2005

\begin{tabular}{|c|c|c|c|c|c|}
\hline Año & Total nacimientos & $\mathrm{RN}$ afectados & Tasa Mil & $\bar{x}$ EM Down & $\overline{\mathrm{x}}$ EM total madres \\
\hline 1972 & 12.530 & 13 & 1,03 & & 25,00 \\
\hline 1973 & 11.153 & 15 & 1,34 & & 24,83 \\
\hline 1974 & 11.043 & 16 & 1,44 & & 25,05 \\
\hline 1975 & 7.393 & 13 & 1,75 & & 25,20 \\
\hline 1976 & 6.998 & 9 & 1,28 & & 25,05 \\
\hline 1977 & 5.780 & 11 & 1,90 & 32,5 & 24,39 \\
\hline 1978 & 2.920 & 8 & 2,74 & 30,5 & 25,37 \\
\hline 1979 & 3.662 & 5 & 1,36 & 28,4 & 24,97 \\
\hline 1980 & 4.043 & 10 & 2,47 & 29,8 & 24,99 \\
\hline 1981 & 4.176 & 9 & 2,15 & 31,3 & 25,24 \\
\hline 1982 & 3.804 & 7 & 1,84 & 33,0 & 25,51 \\
\hline 1983 & 4.519 & 8 & 1,77 & 29,1 & 25,93 \\
\hline 1984 & 3.889 & 5 & 1,29 & 28,0 & 26,16 \\
\hline 1985 & 4.321 & 7 & 1,62 & 29,9 & 26,10 \\
\hline 1986 & 3.665 & 2 & 0,65 & 34,1 & 26,20 \\
\hline 1987 & 3.739 & 6 & 1,60 & 29,7 & 27,08 \\
\hline 1988 & 2.961 & 7 & 2,36 & 32,2 & 27,38 \\
\hline 1989 & 2.820 & 7 & 2,48 & 33,9 & 27,16 \\
\hline 1990 & 3.748 & 8 & 2,1 & 28,8 & 26,77 \\
\hline 1991 & 4.160 & 9 & 2,16 & 34,0 & 26,50 \\
\hline 1992 & 3.416 & 10 & 2,93 & 39,9 & 26,52 \\
\hline 1993 & 2.281 & 8 & 3,07 & 30,5 & 27,05 \\
\hline 1994 & 2.56 & 5 & 1,96 & 36,5 & 27,29 \\
\hline 1995 & 2.650 & 3 & 1,13 & 34,3 & 27,80 \\
\hline 1996 & 2.355 & 5 & 2,12 & 32,0 & 28,20 \\
\hline 1997 & 2.485 & 7 & 2,82 & 36,1 & 28,31 \\
\hline 1998 & 2.917 & 8 & 2,74 & 38,0 & 28,77 \\
\hline 1999 & 2.267 & 4 & 1,76 & 33,0 & 28,25 \\
\hline 2000 & 2.208 & 6 & 2,71 & 328 & 29,59 \\
\hline 2001 & 2.160 & 6 & 3,70 & 33,0 & 29,37 \\
\hline 2002 & 1.840 & 9 & 5,98 & 33,0 & 29,77 \\
\hline 2003 & 1.584 & 14 & 10,10 & 37,0 & 26,84 \\
\hline 2004 & 1.539 & 7 & 4,55 & 37,9 & 29,26 \\
\hline 2005 & 767 & 4 & 5,22 & 34,5 & 27,77 \\
\hline
\end{tabular}

de síndrome de Down, se observa que ambas aumentan significativamente con pendientes que si bien son diferentes (edad materna promedio: pendiente 0,141 y tasa de síndrome de Down pendiente 0,11), esta diferencia no alcanza la significación estadística $(\mathrm{t}=1,12 ; \mathrm{p}>0,05)$.
Es importante destacar que a medida que aumenta el promedio de edad materna, aumenta también el riesgo de tener un hijo afectado de síndrome de Down. En efecto, en los grupos etarios entre 20 y 29 años el riesgo fue de alrededor de 1/600 nacimientos, para subir a 1/390,8 en el grupo de 30 a 
Tabla 3. Síndrome de D own: N úmero de pacientes, promedios de peso al nacimiento, promedio edad materna y tasa por 1.000 nacidos vivos. Promedio de edad materna de la población general. 1997-2005. M aternidad H ospital C línico Universidad de C hile

\begin{tabular}{|c|c|c|c|c|c|}
\hline \multirow[b]{2}{*}{ Años } & \multicolumn{4}{|c|}{ Síndrome de Down } & \multirow{2}{*}{$\begin{array}{c}\text { Controles } \\
\text { Prom. edad materna }\end{array}$} \\
\hline & Pacientes & Prom. peso (gr) & Prom. edad materna & Tasa/oo & \\
\hline 1997 & 7 & $3.077,14$ & 36,14 & 2,82 & 27,08 \\
\hline 1998 & 8 & $3.451,25$ & 38 & 2,74 & 28,77 \\
\hline 1999 & 4 & 2.545 & 33 & 1,76 & 28,25 \\
\hline 2000 & 6 & 3.202 & 32,8 & 2,71 & 29,59 \\
\hline 2001 & 6 & $3.461,42$ & 33 & 2,77 & 29,67 \\
\hline 2002 & 9 & $2.751,25$ & 33 & 4,89 & 29,77 \\
\hline 2003 & 14 & $2.787,81$ & 37 & 8,19 & 26,84 \\
\hline 2004 & 7 & $3.233,57$ & 37,9 & 4,50 & 29,26 \\
\hline 2005 & 4 & 2.375 & 34,5 & 5,23 & 27,77 \\
\hline Total & 65 & 3.010 & $35,5^{*}$ & 3,36 & 28,81 \\
\hline
\end{tabular}

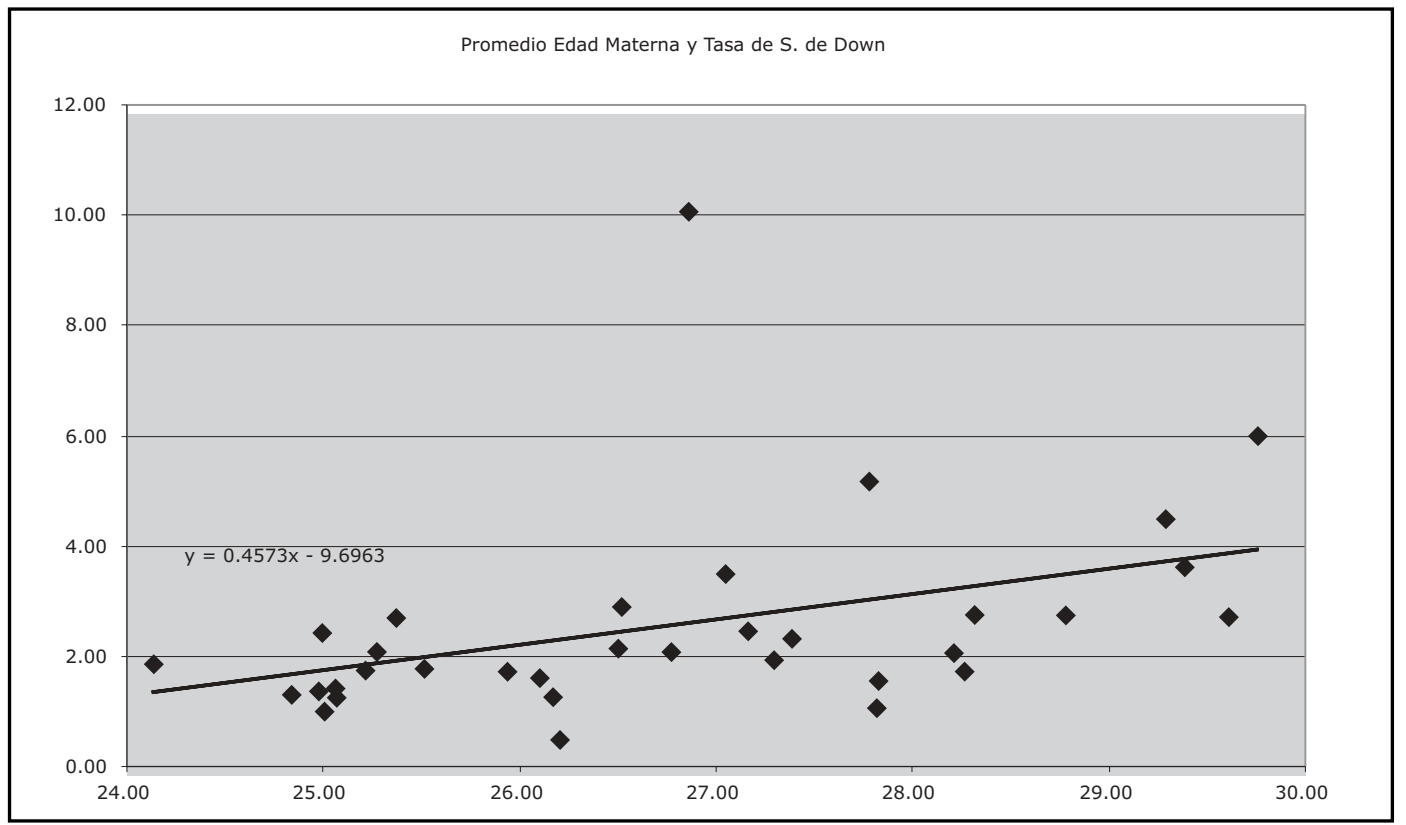

Figura 2. Recta de regresión lineal de la tasa anual por mil de prevalencia de síndrome de Down en relación al promedio de edad materna. Período 1972 a 2005.

34 años, a 1/164,1 entre 35 y 39 años y sobre los 39 años alcanzar la impresionante cifra de 1/33,1 nacimientos. El riesgo global fue de 1/257,2 (Tabla 4).

Cuando comparamos las tasas de prevalencia al nacimiento de los distintos hospitales chilenos participantes en el ECLAMC (Tabla 5), podemos apreciar una gran homogeneidad entre ellos. La tasa global fue de 1,96 por 1.000 nacimientos. La gran mayoría de los hospitales con un gran número de nacimientos, tenían tasas sobre 2 por 1.000, el total del ECLAMC tiene 1,63 por mil, el resto del ECLAMC (sin Chile), 1,59 por 1.000. La 
Tabla 4. Total de nacidos vivos, número de casos con síndrome de D own y riesgo por grupo etario. M aternidad del H ospital C línico U niversidad de C hile. Período 1997-2005

\begin{tabular}{|lrrr|}
\hline Grupo etario & Nacidos vivos & S. De Down & Riesgo \\
\hline$<15$ años & 38 & 0 & 0 \\
15 a 19 & 1.022 & 0 & 0 \\
20 a 24 & 3.119 & 5 & $1 / 623,8$ \\
25 a 29 & 5.114 & 9 & $1 / 568,2$ \\
30 a 34 & 4.299 & 11 & $1 / 390,8$ \\
35 a 39 & 2.461 & 15 & $1 / 164,1$ \\
$>39$ & 662 & 20 & $1 / 33,1$ \\
Total & 16.715 & 65 & $1 / 257,2$ \\
\hline
\end{tabular}

Tabla 5. N úmero de nacimientos, número de pacientes con síndrome de $\mathrm{D}$ own y tasas por mil en los hospitales chilenos participantes en el EC LAM C, total de C hile, total del EC LAM C y resto de EC LAM C (sin C hile). Período 1982-2001

\begin{tabular}{|llrrr|}
\hline Hospital & Ciudad & Nacimientos & S. Down & Tasa/mil \\
\hline Clín. U. Chile & Santiago & 63.656 & 128 & 2,01 \\
Clín. C. Freire & Santiago & 3.942 & 4 & 1,01 \\
Dr. Juan Noé & Arica & 3.419 & 5 & 1,46 \\
Regional & Valdivia & 46.437 & 88 & 1,90 \\
Higueras & Talcahuano & 76.566 & 139 & 1,82 \\
Clín. Las Condes & Santiago & 14.846 & 24 & 1,62 \\
Militar & Santiago & 9.845 & 11 & 1,12 \\
Grant Benavente & Concepción & 53.344 & 83 & 1,56 \\
Naval & Valparaíso & 13.246 & 33 & 2,49 \\
Clín. Francesa & Concepción & 6.417 & 15 & 2,34 \\
Gustavo Fricke & Viña del Mar & 10.623 & 22 & 2,07 \\
Clín. S. Alemán & Valdivia & 9.257 & 21 & 2,27 \\
Clín. Tabancura & Santiago & 1.581 & 3 & 1,90 \\
Del Profesor & Santiago & 1.169 & 2 & 1,71 \\
Regional & Rancagua & 22.399 & 60 & 2,68 \\
Dr. E. Torres & Iquique & 2.046 & 3 & 1,47 \\
Hospital Base & Linares & 5.753 & 15 & 2,61 \\
Hospital de & Puerto Montt & 5.944 & 10 & 1,68 \\
Sótero del Río & Santiago & 15.331 & 38 & 2,48 \\
Salvador & Santiago & 12.129 & 33 & 2,72 \\
San Borja Arriarán & Santiago & 13.474 & 35 & 2,60 \\
San José & Santiago & 15.260 & 34 & 2,23 \\
Regional & Talca & 4.087 & 4 & 0,98 \\
Hospital de & Cauquenes & 349 & 0 & 0,00 \\
Hospital de & Curicó & 1.048 & 3 & 2,86 \\
Total de Chile & & 412.168 & & 1,96 \\
Resto del ECLAMC & & 3.162 .441 & & 1,59 \\
& & & & \\
\hline
\end{tabular}


cifra más alta la tuvo el Hospital del Salvador con 2,72 por 1.000, seguido del Hospital San Borja Arriarán con 2,60 por 1.000, el Hospital Sótero del Río con 2,48 y el HCUCH 2,01 por 1.000 .

$\mathrm{Si}$ agrupamos los hospitales por zonas geográfcas, Sur, Norte, Quinta Región y Santiago, observamos que existen diferencias estadísticamente significativas entre las regiones $\left(\chi^{2}=18,15\right.$ con 3 grados de libertad; $\mathrm{p}=0,0004$ ) debido a la alta tasa de Santiago y a la baja frecuencia en la zona sur y norte del país (Tabla 6).

El promedio de peso de nacimiento para los $\mathrm{RN}$ con síndrome de Down fue de 3.009,76 gramos (desviación estándar 645,8) y de 3.420,55 gramos (desviación estándar 529,9) para los controles ( $\mathrm{t}=4,79 ; \mathrm{p}<0,001)$.

Revisados los casos de síndrome de Down a los que se le había realizado cariograma (61/65), en sólo $3(4,9 \%)$ se encontró translocación, lo que es concordante con lo descrito por otros autores ${ }^{9-11}$.

\section{DisCUSIÓN}

La tasa de prevalencia al nacimiento de síndrome de Down de 3,36 por mil, encontrada para el período 1997-2005, fue significativamente más alta que las tasas encontradas por nosotros en estudios anteriores realizados en la maternidad del Hospital Clínico de la Universidad de Chile. En efecto, en el período 1972-19773, la prevalencia al nacimiento de síndrome de Down fue de 1,39 por mil nacidos vivos, aumentó a 1,74 por mil en el período $1977-1989^{5}$, a 2,35 por mil entre 1990 y $1997^{4}$ y a 3,36 por mil en esta última muestra. En la Tabla 2, y Figura 1, se muestra en forma detallada, cómo a través de los años, esta tendencia al alza de las frecuencias se ha ido consolidan- do en cifras sobre 5 por mil, lo que es realmente preocupante.

¿Cómo explicar este fenómeno que estamos observando? No se puede afirmar que por ser un hospital de referencia para muchas patologías maternas y fetales, nazcan más niños afectados por el síndrome de Down, especialmente porque no es un diagnóstico que se haga en forma rutinaria por exámenes prenatales. Es posible sospecharlo cuando, por la ecografía prenatal, se encuentra translucencia retronucal o atresia de duodeno, pero es cierto también que muchas mujeres prefieren no saberlo y están en su derecho a negarse a practicarse exámenes complementarios. Un hecho que se ha documentado como factor de riesgo para tener hijos con esta anomalía, es el aumento de la edad materna $^{5}$. En la maternidad del $\mathrm{HCUCH}$, el promedio de la edad matema ha ido incrementándose desde alrededor de 23 años en la década de 197079, a 26 en los años 1980-89, 28 en 1990-99 y sobre 29 en la actualidad (Tabla 2). Este aumento es estadísticamente significativo. La relación de estos dos incrementos, tasa de síndrome de Down y promedio de edad materna, se muestran en las Figuras 1 y 2.

El promedio de edad materna de los pacientes afectados fue de 35,5 años (desviación estándar 6,46 años), contra 28,81 años (desviación estándar 6,08 años) de las madres de niños controles sin malformaciones congénitas ( $t=7,6$; $p=0,0001$ ).

En nuestra muestra, 18,7\% de las mujeres tenían 35 años o más y fueron las madres de $53,8 \%$ de los niños afectados. Las mujeres de 40 años o más constituían $3 \%$ del total y fueron madres de $30,8 \%$ de los niños con síndrome de Down. Una medida de prevención primaria de máxima importancia, sería desincentivar a las

Tabla 6. D istribución por zonas de los casos de síndrome de D own de los hospitales chilenos del EC LAM C. Período 1982-2001

\begin{tabular}{|lrrr|}
\hline Zona & № nacimientos & S.Down & Tasa por mil \\
\hline Santiago & 151.233 & 380 & 2,51 \\
Quinta Región & 23.869 & 55 & 2,30 \\
Sur & 231.592 & 438 & 1,89 \\
Norte & 5.565 & 8 & 1,46 \\
\hline
\end{tabular}

$\chi^{2}=18,15$ con 3 grados de libertad; $p=0,000$. 
mujeres de esos grupos etarios a que se embaracen. Es una medida de salud pública de bajo costo, que sólo necesita de educación a las parejas, que deben ser informadas de los riesgos, lo que evitaría un gran número de niños afectados. Por desgracia, no es un problema de fácil solución, ya que hay parejas de edades mayores, aún bien informadas, que deciden tener un hijo.

Es interesante hacer notar que el aumento de las tasas de prevalencia al nacimiento de síndrome de Down es muy superior al incremento observado en los promedios de edad materna. La tasa de síndrome de Down casi se ha triplicado en 34 años, de 1,03 por mil nacimientos en 1972 a 5,22 por 1.000 nacimientos en 2005 (Tabla 2), mientras que el promedio de edad materna sólo se ha incrementado en $16 \%$ ( 25 años en 1972 a sobre 29 años desde 2002). Esto estaría demostrando que un pequeño aumento del promedio de la edad

\section{REFERENCIAS}

1. Castili EE, Oriol IM. The Latin American Collaborative Study of Congenital Malformations. Community Genetics 2004; 7: 76-94.

2. Nazer J, Antolini M, Jú́rez ME, Cifuentes L, Hübner ME, Pardo RA ET AL. Prevalencia al nacimiento de aberraciones cromosómicas en el Hospital Clínico de la Universidad de Chile. Período 1990-2001. Rev Méd Chile 2003; 131: 651-58.

3. Nazer J, Eagun MA, Cifuentes L Incidencia del Síndrome de Down en la matemidad del Hospital Clínico de la Universidad de Chile. Un registro de 25 años: 1972-1997. Rev Méd Chile 1998; 126: 383-90.

4. Nazer J, Ruzzardini M, Díaz-Vélis G, Walton R. Malformaciones Congénitas VII: Síndrome de Down. 1971-1977. Pediatría (Santiago) 1980; 23: 82-88.

5. Nazer J, Hübner ME, Cifuentes L, Ramírez R, Catalán J, Ruiz G. Aumento de la incidencia del Síndrome de Down y su posible relación con el incremento materna provocaría un fuerte incremento del riesgo de tener un hijo afectado.

Desde hace tiempo se conoce la estrecha relación que existe entre la edad materna y el riesgo de tener un hijo con alguna trisomía, pero desconocemos los mecanismos responsables de esta asociación. No hay acuerdo sobre ello. Una posible alternativa sería que los embriones trisómicos tendrían una sobrevida mayor en las mujeres de mayor edad, debido a una disminución de la capacidad inmunológica, por lo que no se abortarían ${ }^{12}$. Está, además, la antigua teoría de los 〈óvulos viejos»0 〈envejecimiento ovular», ya que la mujer nace con una población fija de oocitos y que a medida que va pasando el tiempo, se producirían fallas en la separación de los cromosomas durante la meiosis materna ${ }^{13}$. Se acepta además que la edad paterna no constituye riesgo para tener un hijo con trisomía ${ }^{5}$.

de la edad materna. Rev Méd Chile 1991; 119: 465-71.

6. NAZER J. Anomalías congénitas: Prevención primaria. Rev Hospital Clínico Universidad de Chile 2002; 13 (4); 294-305.

7. ECLAMC: Manual Operacional. 2002. Edición electrónica: http://eclamc.fiocruz.ioc.br

8. International Clearinghouse for birth defects Monitoring Systems. Anual report. 1993. Publishes by the ICBD. Roma. Italy.

9. Astete CP. Síndrome de Down. Rev Chil Pediatr 1993; 64 (supl. 1): 18-20.

10. Giraud F, Mattei JF. Aspects epidemiologiques de la trisomie 21. J Génét Human 1975; 23: 1-30.

11. Ноок EG. Epidemiology of Down syndrome. Advances in Biomedicine and the Behavioral Sciences. Cambridge Press 1982; p 11.

12. Mueler RF, Young ID. Emery's elements of medical genetics. $9^{\text {th }}$ edition. Churchill Livingtone. 1995.

13. Thompson and Thompson. Genetics in medicine. $4^{\text {th }}$ Edition WB Saunders Company. 1986; 126-29. 